\title{
Subject Index
}

Accessory flexor digitorum muscle 62

- $\quad$ nerve 124,150

Amphioxus 96

Apocrine secretion 8

Atresia 189

Atrioventricular septum 27 Axial specification 110

Basal cells 181 Bats 219 Brainstem 124 Branches 57 Branchiomotor 132

Caecilians 160

Cell contacts 169

Cervical spinal cord 124

Chemical senses 132

Chick 27

Chondrosarcoma 1

Clomiphene citrate 14

Comparative anatomy 124

Connective tissue 197

Cranial nerves 96, 110, 124, 150, 160

Cytoarchitecture 213

Cytoplasmic organdies 181

Density 206 Development 96 Digital lock 219 Discordancy 176

Efferent neurons 110 Elastic fibers 197 Electron microscopy 34 Estrous cycle 189 Evolution 96

Evolutionary morphology 124

Fabrica 228 Facial nerve 42 Female dog 197 Fibre number 22

- $\quad$ size 22

FMRFamide 81

Follicle 189

Functional components 132

Gene expression 110 Gerbil 189

Gonadotropin-releasing hormone 81 Growth retardation 176 Gymnophiona 160

Hanging mechanisms 219 Head segmentation 71 Heart 27 Hindbrain 110 Hindlimbs 219 History of anatomy 228 Horseradish peroxidase 42 Human(s) 27, 57 - development 169 Hypoglossal nerve 124,160

Immunocytochemistry 34 Infraorbital gland 8 Interhyoideus posterior 160 Internal carotid artery 57 Intracavernous course 57

Japanese serow 8

Lampreys 96

Luteinizing hormone-releasing hormone 81 
Mandibular musculature 139 Mesencephalic trigeminal nucleus 34 Mesoscopic anatomy 57 Microleaflets 27 Morphometric study 42 Motor nuclei 124 Muscle development, prenatal 22 Musculus flexor digitorum 62

Nervus terminalis 81 Neural crest 71 Neuromeres 71, 110 Node distribution 206 Nucleus olfactoretinalis 81

Olfaction 132 Olfactory system 81 Ontogeny 139 Ovary 189

Phenobarbital, prenatal 22 Phylogeny 139 Pituitary gland 169 Placodes 71

Quantitative bone histomorphometry 1

Rat $14,34,206$

- $\quad$ facial nucleus 42

Rathke's pouch 169

Reproduction 81

Retinacula 219

Rhombomeres 110

Right atrioventricular orifice 27

Scanning electron microscopy 8, 49, 213

Secretory cells 181

Seminal vesicle 169

Serial homology 71

Serotonin 34

Smooth musculature 197

Somitomeres 71, 110

Spinal nerve(s) 96,160,206

Stereology 197

Superior cervical ganglion 49,213

Tarsal tunnel 62

Taste 132

Tendons 219

Tentacle 160

Terminal nerve 81

Toe 62

Topographical organization 42

Transitional zone 206

Tree shrew 49, 213

Trigeminal nerve 139

Twins 176

Ultrastructure 1 Urethra 197 Urethral sphincter 197 Urinary continence 197

Vagina 14

Vaginal epithelium 14 Vagus nerve 124, 150 Vascular cast 49

plexus 197 Vertebrate(s) 124

evolution 110 Vesalius 228 